\title{
Optical anisotropy induced by ion bombardment of $\operatorname{Ag}(001)$
}

\author{
Frank Everts, Herbert Wormeester, and Bene Poelsema \\ MESA + Research Institute, University of Twente, P. O. Box 217, 7500 AE Enschede, The Netherlands
}

(Received 19 May 2008; revised manuscript received 6 August 2008; published 15 October 2008)

\begin{abstract}
Grazing incidence ion bombardment results in the formation of nanoripples that induce an anisotropic optical reflection The evolution of the reflectance anisotropy has been monitored in situ with reflectance anisotropy spectroscopy. The Rayleigh-Rice theory (RRT) has been used to analyze the optical spectra quantitatively and provides the evolution of the average ripple period and root-mean-squared surface roughness. After an incipient phase, both the increase in the periodicity and the roughness vary roughly with the square root of the sputter time. Additional high-resolution low-energy electron diffraction (HR-LEED) measurements have been performed to characterize details of the average structure created by ion bombardment.
\end{abstract}

DOI: 10.1103/PhysRevB.78.155419 PACS number(s): 79.20.Rf, 81.16.Rf, 61.05.jh, 73.20.Mf

\section{INTRODUCTION}

In the last decade, ion beam erosion has emerged as a versatile technique for the creation of nanopatterns. ${ }^{1-6}$ This technique has great potential since it enables a fast and easy way to create large homogeneous areas with highly ordered features. Most common is the formation of a pattern with a height modulation in one direction, a nanoripple pattern. After sufficient ion fluence during off-normal ion bombardment, a stationary situation results, characterized by the periodicity of the nanoripples. The latter is determined by the combination of the diffusive properties of species on the surface, the polar angle of incidence, and the incident ion flux, mass, and energy. ${ }^{7,8}$ This periodicity in the stationary situation has been observed on many surfaces, among them the crystalline $\mathrm{Cu}(001)$ surface. ${ }^{1,9}$ This evolution has been compared to the aeolus evolution of sand dunes by Aste and Valbusa. ${ }^{10}$ They explained the evolution of the ripple structure with the variation in erosion amplitude of various wavelengths present on the surface that are triggered by random fluctuations. However, the evolution of the periodicity and surface roughness before the stationary situation is achieved has only been the subject of a limited number of experimental studies. ${ }^{1-14}$ In situ experimental investigations of the ripple evolution are hampered by the ion beam used in the erosion process. It requires gas pressures and geometries that are not compatible with many microscopy and diffraction techniques. In this article we will show that optical metrology provides an excellent method for in situ characterization of the average ripple period, the surface roughness, and their evolution under these circumstances.

Optical characterization of ripple formation on the $\mathrm{Cu}(001)$ surface by ion beam erosion was already performed by Chan et al. ${ }^{9}$ With light scattering spectroscopy (LiSSp) they were able to characterize the periodicity of ripples with an average periodicity between 300 and $2000 \mathrm{~nm}$. However, the LiSSp method detects light scattered at angles different from the specular beam. This implies that at least two viewports are required and their orientation limits the periodicities that can be measured. Furthermore, it relies on the detection of a weak signal, while it is also not sensitive to roughness below the diffraction limit. Reflective anisotropy spectroscopy (RAS) is a technique that can overcome these restrictions. It was introduced by Aspnes et al. ${ }^{15,16}$ to study the above-band-gap anisotropy of cubic semiconductors and has matured in a versatile technique for the analysis of optical anisotropy at a surface by reflection of a light beam at normal incidence. ${ }^{17}$ The ripple pattern induced by ion bombardment induces a difference in reflection for light polarized parallel and perpendicular to the ripple pattern. Effective medium theories that describe the dielectric function of a layer as a result of a heterogeneity on a length scale below the diffraction limit often take the specific geometry into account. ${ }^{18}$ The one-dimensional (1D) ripple pattern can be viewed as a lamellar structure. A difference in effective dielectric function parallel and perpendicular to the lamella was already derived by Wien. This implies that RAS is sensitive to anisotropic structures with a periodicity below the diffraction limit. This technique was already employed by Martin et al. ${ }^{19}$ to study ion erosion. They limited their study to the effects of ion bombardment on the optical and electronic properties of the intrinsically anisotropic $\mathrm{Cu}(110)$.

In this work, we present a study of the ripple formation through ion bombardment on the intrinsically isotropic $\operatorname{Ag}(001)$ surface. The ripple formation on the $\operatorname{Ag}(001)$ surface has been studied for two reasons. First, the surface shows no anisotropy before ion sputtering. Any observed anisotropy is therefore directly related to the ion bombardment. With an appropriate analysis, the observed anisotropy can thus be related quantitatively to the average ripple periodicity and root-mean-square (rms) roughness upon ion erosion. The second reason for using this surface is the presence of strong plasmonic effects. The strong coupling between the wavelength of the incident light and the periodic length scale of features on the surface induces an absorption at a photon energy that is very characteristic for the specific ripple period. Therefore, for features of even a few monolayers deep, the optical anisotropy is already significant. This enables the in situ monitoring of the pattern formation directly from the start and makes RAS a sensitive tool for the analysis of surface morphology. The ripple formation has been studied at a polar angle of incidence of the ion beam of $70^{\circ}$ and $80^{\circ}$. Additional electron diffraction experiments reveal that at a polar angle of $70^{\circ}$ the etch structures still show persistent two-dimensional (2D) character, ${ }^{12}$ although the optical measurements are sensitive for the ripple periodicity only and not for the ripple length for the range of temperatures studied. At 


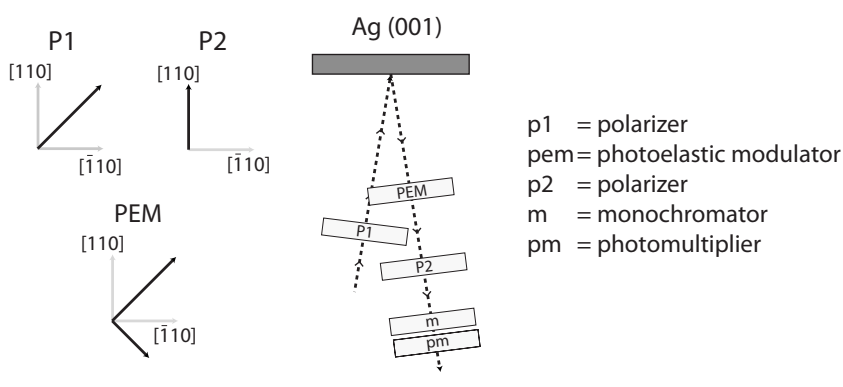

FIG. 1. Schematic overview RAS setup.

$80^{\circ}$ a $1 \mathrm{D}$ ripple pattern is observed with both optical and electron diffraction methods.

\section{EXPERIMENTAL}

The experiments were performed in an ultrahigh vacuum (UHV) chamber with a base pressure below $10^{-10}$ mbar. The cleaning procedure of the $\mathrm{Ag}(001)$ crystal consisted of 45 min of sputtering under an angle of $45^{\circ}$ along the crystallographic $[\overline{1} 10]$ direction and subsequent annealing at $700 \mathrm{~K}$ for $30 \mathrm{~min}$. The cleanliness of the surface was monitored by Auger spectroscopy until no traces of surface contamination could be found, which ensures a contamination level below about one atomic percent. The system was further equipped with an Omicron spot profile analysis low-electron-energy diffraction (SPA-LEED) system. At in-phase conditions, the Bragg peak had a width of about $0.4 \%$ Brillouin zone (BZ), indicating an average terrace width of about $100 \mathrm{~nm}$ without any preferential direction for a freshly prepared sample. This instrument was also used to characterize the structures after ion bombardment.

A homebuilt RAS setup (Fig. 1) was used in our experiments very similar to the one described by Aspnes et al. ${ }^{16} \mathrm{~A}$ Xe light source was used to create a near parallel light beam. This beam was linearly polarized along the crystallographic [010] direction of the substrate before entering the UHV chamber through a strain-free quartz window. The beam was reflected by the substrate at a near normal angle and passed through the same UHV window. The specular reflected beam passed through a photoelastic modulator (type Hinds PEM90) also oriented in [010] with respect to the substrate. With this setup the change in polarization by the surface can be probed. Both the intensities of the first and second harmonic of the modulated signal with respect to the dc intensity are measured with a lock-in technique. This provides both the imaginary and the real part of the reflectance difference, which is defined as

$$
\frac{\Delta R}{R}=2\left(\frac{R_{[110]}-R_{[\overline{110]}}}{R_{[110]}+R_{[110]}^{-}}\right)
$$

with $R_{[110]}$ and $R_{[110]}^{-}$the reflectivity of the $\operatorname{Ag}(001)$ surface along the $[110]$ and $[\overline{1} 10]$ azimuth, respectively. The precision achieved in using this setup amounts to $\Delta R / R=5$ $\times 10^{-5}$, while a spectral range of $226-830 \mathrm{~nm}(1.5-5.5 \mathrm{eV})$ is accessible. A measurement of this complete spectral range takes approximately $18 \mathrm{~min}$.
Grazing incidence ion bombardment along the [ $\overline{1} 10]$ crystallographic direction has been performed with a polar angle of incidence of $\theta_{i}=70^{\circ}$ and $80^{\circ}$ with the surface normal. The energy of the $\mathrm{Ar}^{+}$ions is $2 \mathrm{keV}$ and a flux of $5 \mu \mathrm{A} \mathrm{cm}^{-2} \mathrm{~s}^{-1}$ $\left(3 \times 10^{13}\right.$ ions $\left.\mathrm{cm}^{-2} \mathrm{~s}^{-1}\right)$ has been used. This flux is equivalent to an impingement rate of 1.5 monolayer equivalent (MLE) /min. One MLE is defined as the ion dose required to have a surface atom hit by one incident ion on average. The sputter time in all experiments is $18 \mathrm{~h}$, resulting in a fluence of $2 \times 10^{18}$ ions $\mathrm{cm}^{-2}$. During the ion bombardment, a RAS spectrum is taken every $20 \mathrm{~min}$. The sample temperature is kept constant with an accuracy of $\pm 2 \mathrm{~K}$ in the range of 300 $\mathrm{K}-450 \mathrm{~K}$. Directly after switching off the ion beam, the sample is cooled to below $130 \mathrm{~K}$ to "freeze in" the obtained ripple pattern. High-resolution electron diffraction experiments have been performed at this temperature to obtain additional information about the shape of the created surface features including their facets.

Before ion bombardment, a RAS spectrum is taken of the optically isotropic clean $\operatorname{Ag}(001)$ surface. Weak deviations from zero anisotropy are attributed to systematic errors and used to correct the measurements. By rotating the clean sample by $90^{\circ}$, the sign and strength of the optical anisotropy was very similar, which confirms that this signal can indeed be attributed to systematic errors of the setup.

\section{RESULTS}

The evolution of the RAS spectra during ion bombardment is shown in Figs. 2(a)-2(d) for different sample temperatures for a polar angle of incidence of $70^{\circ}$. The interval between successive spectra is $1 \mathrm{~h}$. The ion bombardment results in a plasmonic feature with a strength increasing with sputter time. For temperatures up to $320 \mathrm{~K}$, the energy position of this plasmonic feature is similar to the surface plasmon energy of $\mathrm{Ag}$, which is about $3.70 \mathrm{eV}$ and only slightly temperature dependent. ${ }^{20}$ The increase in signal strength is a result of a significant roughening of the surface, while the fixed position indicates that the periodicity of this roughness is below $200 \mathrm{~nm}$. For higher temperatures, a different behavior is observed. The increasing signal strength is accompanied by a redshift of the plasmon energy peak and also peak broadening is visible. The redshift indicates an increase in the average distance between ripples. As expected, the ripple periodicity increases during the sputtering. With increasing sample temperature, the lateral dimensions also increase and thus this shift becomes increasingly more distinct. The peak broadening indicates a widening lateral periodicity distribution on the surface. At the low- and high-energy ends of the spectra, the noise level increases due to a decrease in the reflected intensity.

Annealing experiments have also been carried out after the sputter experiments to check the stability of the structures. Without changing the temperature, the change in the RAS spectrum has been monitored after the ion beam has been switched off. After one hour, a decrease of about $3 \%$ of the RAS signal has been found for a sample temperature of $350 \mathrm{~K}$. For a sample temperature of $420 \mathrm{~K}$, a decrease of $20 \%$ has been found. For both temperatures no redshift of the 


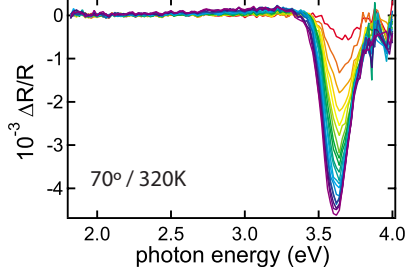

(a)

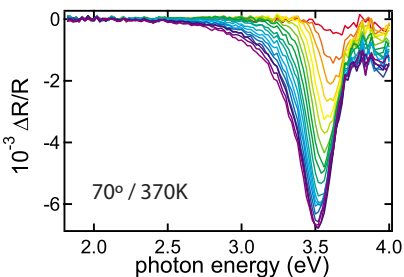

(b)

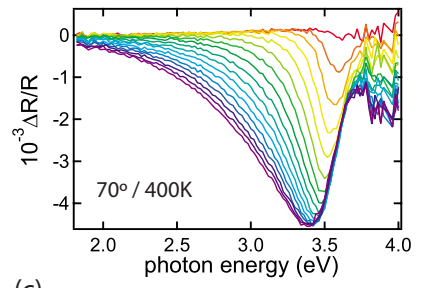

(c)

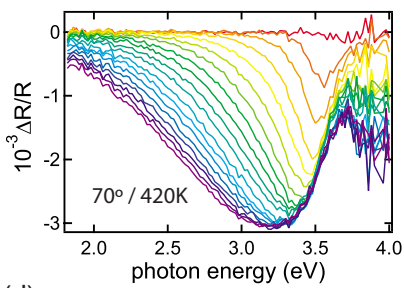

(d)

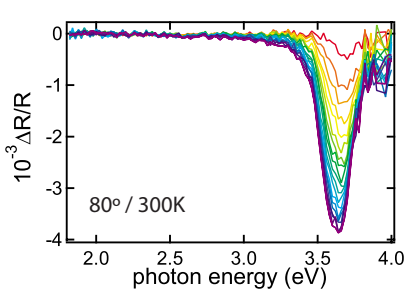

(e)

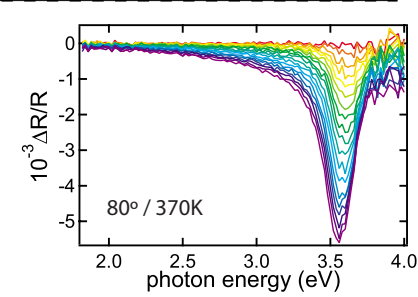

(f)

Sputter time: \begin{tabular}{rl|l|ll|l} 
& &
\end{tabular}

FIG. 2. (Color online) RAS spectra for different sample temperatures and angles of incidence. All samples were bombarded with $2000 \mathrm{eV} \mathrm{Ar}^{+}$ions. For (a)-(d): $\theta_{i}=70^{\circ}$, ion current $j_{i}$ $=5 \mu \mathrm{A} \mathrm{cm}^{-2}$, and sample temperatures $320 \mathrm{~K}, 370 \mathrm{~K}, 400 \mathrm{~K}$, and $420 \mathrm{~K}$, respectively. For (e) and (f): $\theta_{i}=80^{\circ}$, ion current $j_{i}$ $=2 \mu \mathrm{A} \mathrm{cm}^{-2}$, and sample temperatures $300 \mathrm{~K}$ and $370 \mathrm{~K}$, respectively.

feature has been observed, which indicates that the ripple periodicity remains the same at the time scale of the experiment. Since an optical scan takes about $18 \mathrm{~min}$, an error of only a few percent in the RAS signal occurs within one spectrum. The cooling down of the sample to $130 \mathrm{~K}$ after switching off the ion beam only takes a few minutes. The annealing effect during this cooling down is therefore negligible in all cases discussed here.

In Figs. 2(e) and 2(f), the evolution of the RAS spectra during ion bombardment at a polar angle of $\theta_{i}=80^{\circ}$ is shown for two temperatures. The energy position of the features is close to the surface plasmon energy. The average periodicity must therefore be around or below $200 \mathrm{~nm}$. Because of this small ripple periodicity at $80^{\circ}$ incidence, it is not possible to determine the shift in periodicity as a function of temperature and sputter time. The full analysis of the optical spectra is therefore limited to the data taken at $\theta_{i}=70^{\circ}$.

High-resolution low-electron-energy diffraction (HRLEED) measurements reveal that the structures by ion bombardment show well-defined facets as has also been observed for $\mathrm{Cu}(001) .{ }^{12}$ It is not possible to distinguish whether the structures are pits or hillocks with these diffraction measurements. In our analysis we assume dealing with pits. In Fig. 3, the LEED measurements are shown after sputtering at polar angles of incidence of $70^{\circ}$ and $80^{\circ}$. The image after ion
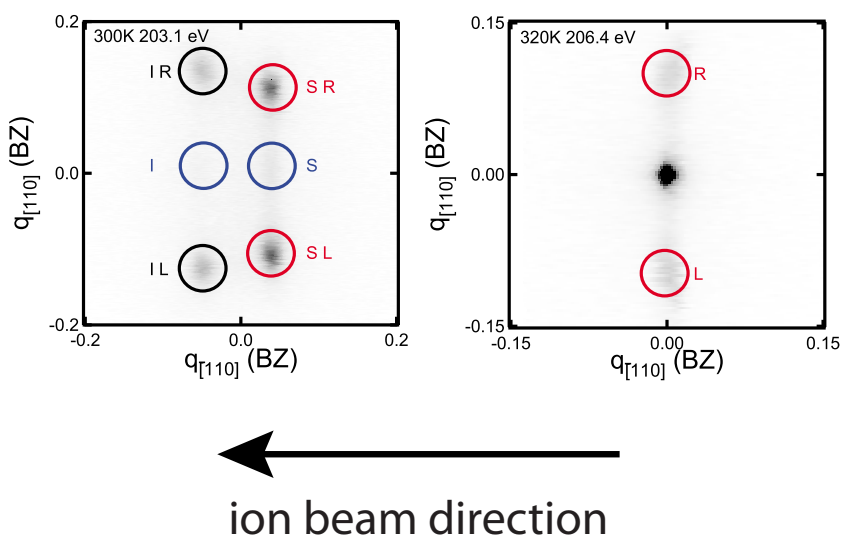

FIG. 3. (Color online) Typical HR-LEED patterns after prolonged sputtering. Both patterns have been obtained under slightly out of phase conditions $\left(S_{[001]}=4.9\right.$; see text $)$. The ion beam was incident from the right. Assuming to deal with pits, the diffraction peaks are identified as resulting from shadow-side $(S)$-and illuminated $(I)$ facets and from left $(L)$-and right-hand side $(R)$ facets. At a polar angle of $70^{\circ}$ (left-hand panel) the pattern is three dimensional (3D) [2D in the (001)-surface plane], while at $80^{\circ}$ (right-hand panel) the pattern is $2 \mathrm{D}$ [1D in the (001) surface plane].

bombardment at a polar angle of incidence of $\theta_{i}=70^{\circ}$ has been recorded at a slightly out-of-phase diffraction condition with $S_{[001]} \approx 4.9$. Note that the perpendicular momentum change $q_{[001]}$ is represented by the phase $S_{[001]}$ relating to $q_{[001]}$ by $q_{[001]}=S_{[001]}(2 \pi / d)$ with $d$ representing the interlayer spacing. Six intensity maxima are identified, three spots at the illuminated side (IR, IL, and I) and three spots at the shadow side (SR, SL, and $S$ ). The pattern exhibits mirror plane symmetry with the mirror plane defined by the plane of incidence of the ion beam. The distance of the intensity maxima with respect to the position of the specular beam $\left(q_{[110]}=q_{[110]}^{-}=0\right)$ increases with increasing $\Delta S=\operatorname{int}(S)-S$, indicating that they arise from facets. This similar diffraction pattern is observed after all erosion experiments, independent of the substrate temperature. At $\theta_{i}=80^{\circ}$, the pattern reduces strictly to only intensity distributed in the plane perpendicular to the plane of incidence of the ion beam, indicating a $1 \mathrm{D}$ ripple pattern. Besides the Bragg peak, only two intensity maxima are identified $(R$ and $L)$ in this case. The length of the ripples is then beyond the resolution of the instrument used, i.e., at least $100 \mathrm{~nm}$. The pattern is again similar for all sample temperatures.

In Fig. 4(a) the positions of the four most pronounced diffraction spots (SR, SL, IR, and IL) are shown as a function of the normalized perpendicular phase $S_{[001]}$ for the $70^{\circ}$ case. Toward the in-phase condition $\left(S_{[001]}=5.0\right)$ the spots move all to the center. This is the position of the $(0,0)$ (Bragg) spot, which is only visible close to the in-phase condition. The small width of the Bragg peak upon varying the perpendicular phase indicates that the structures are at least several layers deep. ${ }^{21,22}$ For increasing $\Delta S$, two weaker side peaks $(S$ and $I)$ appear along the high-symmetry $\left(q_{[110]}\right.$ and $\left.q_{[110]}\right)$ directions. These peaks indicate smaller, less pronounced facets. The orientation of all facets is determined from the displacement of these peaks as function of the perpendicular phases. We find that the displacement in the plane 
(a)

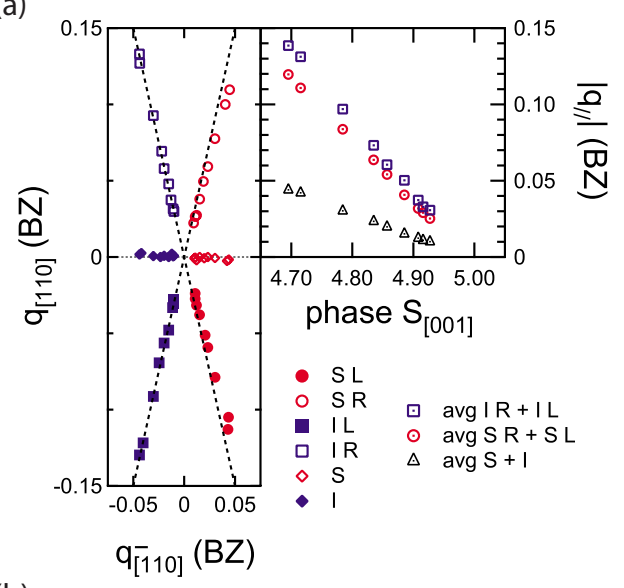

(b)

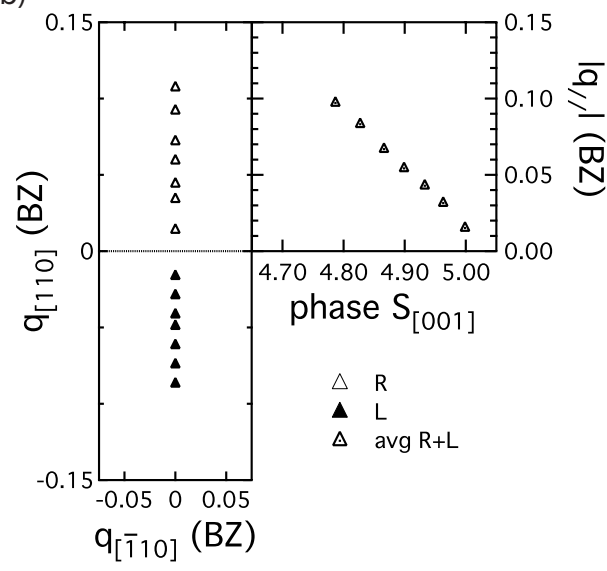

(c)

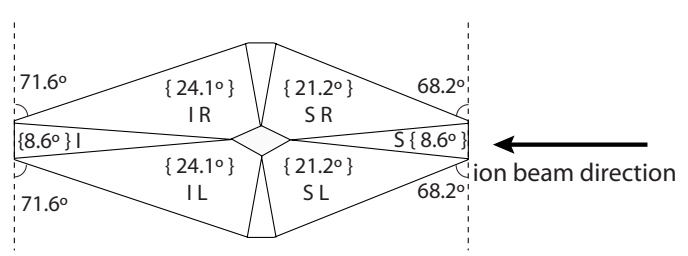

(d)

$\left\{20.8^{\circ}\right\} R$

$\left\{20.8^{\circ}\right\} \mathrm{L}$

FIG. 4. (Color online) Elucidation of typical majority features on $\mathrm{Ag}(001)$ after prolonged bombardment at grazing incidence as revealed by HR-LEED patterns (cf. Fig. 3). Left-hand panels in (a) and (b): Facet peak positions as a function of the changes, $q$, in electron wave vectors along the $[\overline{1} 10]$ and the $[110]$ azimuth for various electron energies between 203.1 and $223.2 \mathrm{eV}$. Right-hand panels of (a) and (b): The average parallel components of the electron wave vector change for the various facets vs the vertical scattering phase $S_{[001]}$ (see text). The surface component of the ions is parallel to $[\overline{1} 10]$ in all cases. The polar angles of incidence are $70^{\circ}$ (a) and $80^{\circ}$ (b) with ion fluences $5 \mu \mathrm{A} / \mathrm{cm}^{2}$ and $2 \mu \mathrm{A} / \mathrm{cm}^{2}$, respectively, and surface temperatures of $300 \mathrm{~K}$ and $320 \mathrm{~K}$, respectively. The corresponding schematic surface features are shown for $70^{\circ}$ in (c) and for $80^{\circ}$ in (d). Assuming as before etch pits the symbols $I$ and $S$ refer to facets on the illuminated and the shadow sides, while $L$ and $R$ refer to left hand and right hand as seen by the incident ions. parallel to the surface is slightly different for the facets on the illuminated and shadow side of the erosion pit. The spots on illuminated side (IR and IL) have an angle of $71.6^{\circ}$ with the high-symmetry $[\overline{1} 10]$ direction. This corresponds to step edges in this facet with a [130] orientation. The angle of the spots on shadow side (SR and SL) has an angle of $68.2^{\circ}$ with respect to the high-symmetry [ $\overline{1} 10]$ direction. This corresponds to a [250] direction of the step edges in this facet. The diffraction features on the shadow side have a significantly stronger intensity. The facet on the incident side of the etch structure has better developed. This is probably the result of the local incidence angle of the ion beam on the facets on the shadow side, which is below $15^{\circ}$ with the local interface. The local incidence angle is then below the critical angle for sputtering. ${ }^{23}$ Below the critical angle, the sputter yield decreases dramatically as most ions are reflected from the surface without an energy transfer impact. However, any protrusion on these facets are very likely to be sputtered away by the ion that skims over the surface for a local incidence angle below the critical angle. ${ }^{24}$ A very efficient mechanism for the creation of well-defined facets is thus obtained. In Fig. 4(b) the positions of the diffraction spots are shown as a function of the normalized perpendicular phase $S_{[001]}$ for the $80^{\circ}$ case. The pattern is in this case one dimensional and only two facets are present. The diffraction spots move along the high-symmetry [110] direction, indicating that the facets are orientated along the sputter direction.

On the right side of Figs. 4(a) and 4(b), the parallel displacement $\left(\left|q_{\|}\right|=\sqrt{q_{[110]}^{2}+q_{[\overline{1} 10]}^{2}}\right)$ of the facet peaks with respect to the origin is plotted as function of the phase $S_{[001]}$. In the $70^{\circ}$ case, a different facet angle has been found for the facets on the illuminated and shadow side of the erosion pit. A facet angle of $21.2^{\circ}$ is found for the shadow facets (SR, $\mathrm{SL}$ ) and a facet angle of $24.1^{\circ}$ is found for the illuminated facets (IR, IL). Also shown are the displacements with perpendicular phase of the less pronounced side peaks $(I, S)$. They reveal facets with an angle of $8.6^{\circ}$ with the surface. After sputtering at a polar angle of $80^{\circ}$, the facet angles of the ripple walls are $20.8^{\circ}(R, L)$. Figures $4(\mathrm{c})$ and $4(\mathrm{~d})$ summarize these angles and show the contours of the average ion erosion pit for both cases.

\section{QUANTITATIVE ANALYSIS OF THE OPTICAL SPECTRA}

The Rayleigh-Rice Theory (RRT) has extensively been used to quantify surface roughness as measured with ellipsometry (see Ref. 25 and references therein). This approach is applicable for relatively small variations on the surface, i.e., $\sigma / \lambda \lesssim 0.05$ and $\sigma / L \cong 0.3$, where $\sigma$ is the rms roughness of the surface, $\lambda$ is the wavelength of the incident light, and $L$ is the characteristic length scale of the surface roughness. The measured surface roughness in our experiments is of the order of a nanometer and is well within the limits for using the RRT analysis.

The RRT is a perturbation approach for the solution of the Maxwell equations on rough surfaces. This was extensively described by Ohlídahl and co-workers ${ }^{25}$ and is given in Eq. 

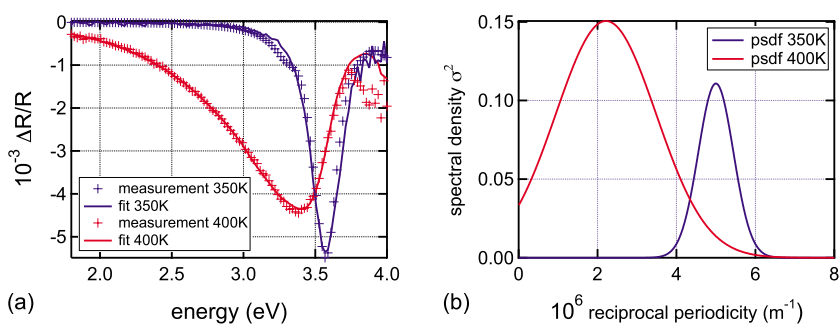

FIG. 5. (Color online) (a) The measured reflectance difference spectra data is indicated with markers. Data is obtained after $18 \mathrm{~h}$ of sputtering with a polar angle of incidence of the ion beam of $\theta_{i}$ $=70^{\circ}$ and sample temperatures of 350 and $400 \mathrm{~K}$. The modeled data is indicated with solid lines and based on a Gaussian distributed PSDF. (b) Gaussian-shaped power spectral density functions used to obtain the modeled spectra in (a).

(2). The zeroth order term $\left(\hat{r}_{j}^{(0)}\right)$ is the Fresnel reflection coefficient for reflection on a smooth surface with polarization $j$. The perturbation term consists of the convolution between the optical response function $\hat{f}_{j}\left(K_{[110]}^{-}, K_{[110]}, k_{0}\right)$ and the normalized power spectral density function (NPSDF) $w\left(K_{[\overline{1} 10]}^{-}\right.$ $\left.-k_{0} \sin \left(\theta_{0}\right), K_{[110]}\right)$, where $k_{0}$ is the wave vector of the incident light at a polar incidence angle of $\theta_{0}$ and $K_{[110]}$ and $K_{[110]}$ are the reciprocal lattice vectors parallel to the surface, which are used to describe the periodicity of the roughness in reciprocal space:

$$
\begin{aligned}
\hat{r}_{j}= & \hat{r}_{j}^{(0)}+\sigma^{2} \int_{-\infty}^{+\infty} \int_{-\infty}^{+\infty} \hat{f}_{j}\left(K_{[\overline{1} 10]}, K_{[110]}, k_{0}\right) \\
& \times w\left(K_{[\overline{1} 10]}^{-}-n_{0} k_{0} \sin \left(\theta_{0}\right), K_{[110]}\right) d K_{[110]}^{-} d K_{[110]} .
\end{aligned}
$$

The rather complex optical response function $\hat{f}$ is simplified by measuring at (near) normal incidence $\left(\theta_{0} \approx 0\right)$. The surface structures are further considered to be one dimensional. This reduces the $K$ vector along the ion beam direction to zero and thus integration over $K_{[110]}$ vanishes. Under these circumstances the anisotropic optical reflection can be described by Eq. (3), where $\epsilon$ is the complex dielectric function of silver. The representation of the NPSDF by a single Gaussian function is found to provide a sufficient description of the data. The use of multiple Gaussians did not result in a significantly better fit. This implies that the pattern at $70^{\circ}$ behaves optically as a 1D structure with negligible change in optical response parallel to the ripples. For the Gaussian distribution the mean value represents the average periodicity of the pattern while the width represents the spread in this periodicity:

$$
\begin{gathered}
\frac{\Delta R}{R}=\sigma^{2} \int_{0}^{\infty}-2 K_{[110]} k_{0}\left(\frac{\sqrt{\kappa-1}-\sqrt{\kappa \epsilon-1}}{1+\sqrt{\kappa-1} \sqrt{\kappa \epsilon-1}}\right) \\
\times w\left(K_{[110]}^{-1}\right) d K_{[110]}^{-}, \\
\kappa=\left(\frac{k_{0}}{K_{[110]}^{-}}\right)^{2} .
\end{gathered}
$$

Figure 5 shows two typical measurements and the fitted spectra. All measured spectra can be described with similar

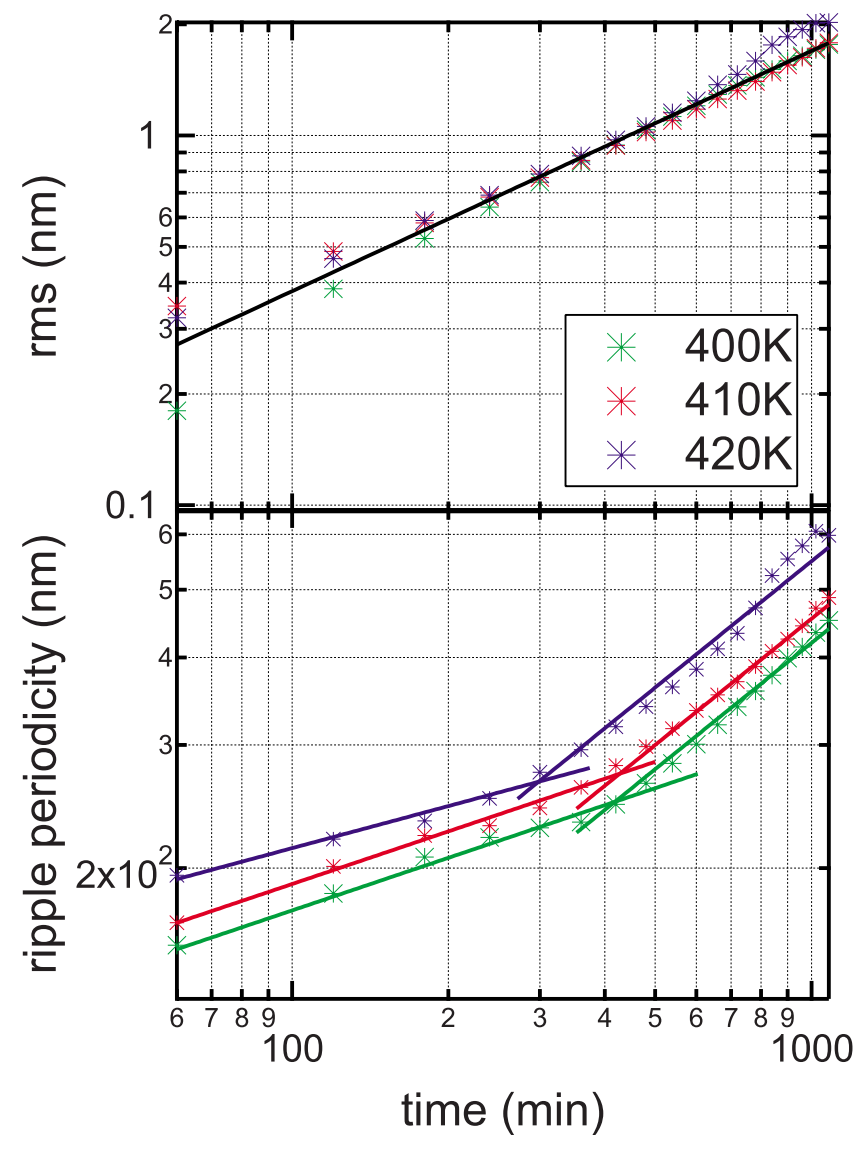

FIG. 6. (Color online) The development of surface rms (top) and the ripple periodicity (bottom) during sputtering as function of time for different sample temperatures. The values have been calculated with the RRT fitting procedure described in the text. The solid lines indicate in the top graph the slope with exponent $\beta$ and in the bottom graph the slope with exponents $p$.

accuracy. Note that for an average periodicity below $200 \mathrm{~nm}$, the plasmon feature remains at the position of the surface plasmon on the silver surface. The RAS technique cannot yield the periodicity although the roughness can be established. The corresponding NPSDFs that are the result of the fit are also shown. We find a considerable broadening of the ripple periodicity with increasing period. The observation of plasmonic features on the Ag surface enables already a direct link between the peak position and the ripple periodicity via the dispersion relation of silver. The maximum position found for the power spectral density function (PSDF) fitted to the reflection difference spectra confirms this relation.

The evolution of ripple periodicity and roughness with sputter time is shown in Fig. 6. In these graphs, only the results for a $70^{\circ}$ polar angle of incidence of the ion beam for the highest substrate temperatures are shown. Only under these conditions, the plasmon features are observed at a position that differs from that of the surface plasmon and the periodicity of the ripple pattern can be determined. The increase with time of the ripple periodicity in the first $300 \mathrm{~min}$ is very different from the later stage. The time of the crossover point decreases with increasing temperature. No saturation of either the average ripple periodicity or the surface roughness is observed. 


\section{DISCUSSION}

Ion bombardment at polar angles of incidence of $70^{\circ}$ or $80^{\circ}$ results in the creation of different structures as indicated by LEED. The most grazing incidence situation $\left(80^{\circ}\right)$ results in the creation of $1 \mathrm{D}$ nanogrooves as already reported by van Dijken et $a l .{ }^{1}$ on the $\mathrm{Cu}(001)$ surface. The intensity of the RAS spectra increases with fluence, indicating that the height of the grooves increases with time in contrast to the lower ion energy results for $\mathrm{Cu}(001)$. The LEED measurements of the deeper grooves on the $\mathrm{Ag}(001)$ surface created by $2 \mathrm{keV}$ $\mathrm{Ar}^{+}$ions show that these grooves indeed have a rectangular profile perpendicular to the ripple direction. The high and low region of the grooves are connected by an edge that consists of a $\{113\}$ facet. At a polar angle of incidence of $70^{\circ}$ the LEED measurements show a limited lateral size of the created structure, i.e., a more $2 \mathrm{D}$ character with an elongated diamond surface pattern. The optical data can, however, still be described well by a 1D optical anisotropy. No sufficient redshift of the plasmon features was noted for sputtering at a substrate temperature below $400 \mathrm{~K}$. This limits the applicability of the RAS method for quantitative analysis under these experimental conditions.

Chan and Chason reported a kinetic phase diagram for pattern formation on $\mathrm{Cu}(001)$ and $\mathrm{Ag}(001)$ surfaces through ion erosion at a polar angle of incidence of $70^{\circ} .^{2}$ As a function of ion current and temperature various regions are identified. At low temperatures, the effectiveness of various interlayer mass transport channels is determined by the associated Ehrlich Schwoebel (ES) barriers. The specific values of these barriers explain the pattern formation on the surface. This is therefore called the ES instability region. At more elevated temperature, the ion flux determines whether roughening is observed. For low flux no roughening is observed while for higher ion flux the pattern formation can be described by the Bradley Harper (BH) approach, the $\mathrm{BH}$ instability region. The results of this work are related to the ES instability region. However, on the basis of the kinetic phase diagram mentioned, the experiments should at least cover two of the regions, as a nonroughening situation is predicted above $380 \mathrm{~K}$. Valbusa et al. ${ }^{3}$ indeed observed rather isotropic structures after $20 \mathrm{~min}$ of $1 \mathrm{keV} \mathrm{Ne}$ ion erosion at $400 \mathrm{~K}$ with an ion current $j_{i}=2.2 \mu \mathrm{A} \mathrm{cm}^{-2}$. However, in the experiments presented in the present work, anisotropic nanopatterns are observed at temperatures as high as $420 \mathrm{~K}$. This is probably due to the 50 times longer sputtering with $2 \mathrm{keV}$ $\mathrm{Ar}^{+}$ions and the higher ion current of $j_{i}=5 \mu \mathrm{A} \mathrm{cm}{ }^{-2}$. After 20 min sputtering we also note only a slight anisotropic nanopattern. This shows that not only ion flux and temperature, but also the fluence is an important parameter for the kinetic phase diagram. The high-resolution LEED images indicate that we do not have elongated ripples as predicted by the Bradley Harper theory. The etch pits show a rather strongly elongated diamond shape. This would imply that these structures fall in the category of nanopatterns that are the result of ES instabilities. The ES barrier of the $\langle 110\rangle$ step edges reduces the path way of interlayer mass transport across these step edges. ${ }^{26}$ Interlayer mass transport via kink positions in the $\langle 110\rangle$ step edge is strongly favored for the $\mathrm{Cu}$ and $\mathrm{Ag}$ (001) surface. ${ }^{6,12,27}$ This explains the observation of the elongated diamond shape as this induces a large number of kinks. For higher ion currents, a transfer to the $\mathrm{BH}$ instability region with the observation of $1 \mathrm{D}$ ripples for a polar angle of incidence of $70^{\circ}$ is expected, in line with reported ripple pattern formation on the $\mathrm{Cu}(001)$ surface. ${ }^{9}$

For elevated temperatures the increase in both periodicity and roughness with time was determined. After an initial period with only a small increase in periodicity, a transition to a stronger increase with fluence is found. However, no saturation of the periodicity has been found, i.e., we obtain no evidence for a stationary situation as described by the Bradley Harper theory. ${ }^{8}$ Recently, Cuerno and co-workers ${ }^{28}$ extended the continuum description of ion erosion processes substantially. The Bradley Harper theory and the more elaborate (anisotropic) Kuramoto-Sivashinsky theory are limiting cases of this description. Also this description allows to characterize the evolution of the ion-induced nanopattern with scaling parameters that describe the change in periodicity and roughness with sputter time. ${ }^{29,30}$ The increase in roughness $\sigma$ with sputter time follows $\sigma \sim t^{\beta}$. The critical exponent $\beta$ has a value of $\approx 0.55$ for the experiments depicted in Fig. 6 . The change in periodicity $L$ is characterized by a critical exponent $p$ with $L \sim t^{p}$. Considering this critical exponent, two stages are identified in our experiments. The transition from the first stage to the second stage is temperature dependent and occurs earlier for higher temperatures. In the initial stage this critical exponent $p$ has a value between 0.2 and 0.25 . These values are similar to the value of around 0.2 found from experiment and theory beyond the KuramotoSivashinsky model. ${ }^{11,13,28}$ However, in the second stage a value for critical exponent $p$ between 0.5 and 0.6 is found. The similarity of $\beta$ and $p$ in this regime indicate that the ratio of height and width of the ripple structures is about constant, independent of fluence and temperature. A value around 0.5 has been reported by Habenicht et al. ${ }^{14}$ for the evolution of the periodicity of ripples created on $\mathrm{Si}(001)$. The evolution of these latter structures has been monitored in situ with SEM enabling to probe not only the periodicity but also the ripple propagation velocity. Also for this experiment, no saturation of the ripple periodicity is observed. This absence of saturation has been explained by the absence of nonlinear terms in the continuum description that would result in a ripple period that grows indefinitely with $p=0.5 \cdot{ }^{28,31} \mathrm{~A}$ similar exponent for the increase in roughness and period is also reported in these simulations. The higher value observed in our experiments might be associated to an efficient coarsening mechanism, ${ }^{28}$ as can be expected for the highly mobile species at temperatures around $400 \mathrm{~K}$.

\section{SUMMARY}

Optical reflection anisotropy is a very suitable tool for the in situ characterization of ion beam induced anisotropic periodic nanopatterns. With the Rayleigh-Rice approximation, the optical response can be quantitatively interpreted in terms of the ripple period, rms, and ordering. Also below the diffraction limit, the formation of a ripple pattern can be observed; albeit the ripple period cannot be established. This limits the quantitative application for the measurement of 
ripple periods on Ag to structures with a characteristic length scale of at least $200 \mathrm{~nm}$. High-resolution LEED data compliments the optical anisotropy observations. For a polar angle of incidence of $80^{\circ}$ a $1 \mathrm{D}$ ripple nanopattern is obtained, while sputtering at $70^{\circ}$ results in anisotropic features, which still have some persistent 2D character. The average shape of the ion pits created can be determined; they have a rather elongated, slightly distorted shape. For the description of the optical response, only the short distance between the etch pits has to be taken into account. A 1D model suffices. Also for extended sputtering time up to $18 \mathrm{~h}$ (fluence 1.9 $\times 10^{18}$ ions $/ \mathrm{cm}^{2}$ ), we do not observe either saturation behavior of the ripple period at temperatures around $400 \mathrm{~K}$ or a saturation of the rms roughness. This indicates that nonlinear mechanisms that are usually observed in ion erosion are not present and coarsening still proceeds in an efficient manner.

\section{ACKNOWLEDGMENT}

This research was supported by NanoNed, a national nanotechnology program coordinated by the Dutch Ministry of Economic Affairs.
${ }^{1}$ S. van Dijken, D. de Bruin, and B. Poelsema, Phys. Rev. Lett. 86, 4608 (2001).

${ }^{2}$ W. L. Chan and E. Chason, J. Appl. Phys. 101, 121301 (2007).

${ }^{3}$ U. Valbusa, C. Boragno, and F. Buatier de Mongeot, J. Phys.: Condens. Matter 14, 8153 (2002).

${ }^{4}$ S. Facsko, T. Dekorsy, C. Koerdt, C. Trappe, H. Kurz, A. Vogt, and H. Hartnagel, Science 285, 1551 (1999).

${ }^{5}$ B. Ziberi, F. Frost, T. Höche, and B. Rauschenbach, Phys. Rev. B 72, 235310 (2005).

${ }^{6}$ P. Broekmann, A. Mewe, H. Wormeester, and B. Poelsema, Phys. Rev. Lett. 89, 146102 (2002).

${ }^{7}$ R. M. Bradley and J. M. E. Harper, J. Vac. Sci. Technol. A 6, 2390 (1988).

${ }^{8}$ R. Cuerno and A.-L. Barabási, Phys. Rev. Lett. 74, 4746 (1995).

${ }^{9}$ W. L. Chan, N. Pavenayotin, and E. Chason, Phys. Rev. B 69, 245413 (2004).

${ }^{10}$ T. Aste and U. Valbusa, New J. Phys. 7, 122 (2005).

${ }^{11}$ S. Rusponi, G. Costantini, C. Boragno, and U. Valbusa, Phys. Rev. Lett. 81, 4184 (1998).

${ }^{12}$ M. M. Ovsyanko, Ph.D. thesis, Twente University, 2006.

${ }^{13}$ T. C. Kim et al., Phys. Rev. Lett. 92, 246104 (2004).

${ }^{14}$ S. Habenicht, K. P. Lieb, J. Koch, and A. D. Wieck, Phys. Rev. B 65, 115327 (2002).

${ }^{15}$ D. Aspnes and A. Studna, Phys. Rev. Lett. 54, 1956 (1985).

${ }^{16}$ D. E. Aspnes, J. P. Harbison, A. A. Studna, and L. T. Florez, J. Vac. Sci. Technol. A 6, 1327 (1988).

${ }^{17}$ P. Weightman, D. S. Martin, R. J. Cole, and T. Farrell, Rep. Prog.
Phys. 68, 1251 (2005).

${ }^{18}$ D. A. G. Bruggeman, Ann. Phys. 24, 636 (1935).

${ }^{19}$ D. S. Martin, R. J. Cole, and P. Weightman, Phys. Rev. B 72, 035408 (2005).

${ }^{20}$ M. Rocca, F. Moresco, and U. Valbusa, Phys. Rev. B 45, 1399 (1992).

${ }^{21}$ J. Wollschläger, E. Z. Luo, and M. Henzler, Phys. Rev. B 57, 15541 (1998)

${ }^{22}$ M. Horn-von Hoegen, Z. Kristallogr. 214, 591 (1999).

${ }^{23}$ H. Niehus, W. Heiland, and E. Taglauer, Surf. Sci. Rep. 17, 213 (1993).

${ }^{24}$ B. Poelsema, L. K. Verhey, and A. L. Boers, Surf. Sci. 55, 445 (1976).

${ }^{25}$ D. Franta and I. Ohlídal, Opt. Commun. 248, 459 (2005).

${ }^{26}$ J. W. Evans, P. A. Thiel, and M. C. Bartelt, Surf. Sci. Rep. 61, 1 (2006).

${ }^{27}$ J. K. Zuo and J. F. Wendelken, Phys. Rev. Lett. 78, 2791 (1997).

${ }^{28}$ J. Munoz-Garcia, M. Castro, and R. Cuerno, Phys. Rev. Lett. 96, 086101 (2006)

${ }^{29}$ A. L. Barabási and H. E. Stanley, Fractal Concepts in Surface Growth (Cambridge University Press, Cambridge, 1995).

${ }^{30}$ Y. Zhao, G.-C. Wang, and T.-M. Lu, Characterization of Amorphous and Crystalline Rough Surface: Principles and Applications, Experimental Methods in Physical Sciences Vol. 37 (Academic, New York, 2001).

${ }^{31}$ M. Raible, S. J. Linz, and P. Hänggi, Phys. Rev. E 62, 1691 (2000). 